\title{
DESEMPENHO DISCENTE DE ENFERMAGEM NA ADMINISTRAÇÃO DE TERAPÊUTICA VIA INTRAMUSCULAR 1
}

\author{
NURSING STUDENTS PERFORMANCE IN ADMINISTERING INTRAMUSCULAR \\ THERAPEUTICS.
}

\author{
Maria Suely Nogueira ${ }^{2}$ \\ Isabel Amélia Costa Mendes ${ }^{3}$ \\ Miyeko Hayashida ${ }^{4}$ \\ Simone de Godoy ${ }^{5}$
}

\begin{abstract}
RESUMO: Em estudo experimental desenvolvido junto a alunos matriculados no curso de graduação em enfermagem, que teve como objetivo comparar o desempenho de dois grupos de alunos, submetidos a dois métodos de ensino diferentes, avaliou-se o desempenho dos mesmos durante sua primeira experiência realizando a aplicação de injetáveis via intramuscular, em situação real, onde o aluno realizou o procedimento em um cliente que procurava a Unidade Básica de Saúde, com a apresentação de receita médica que justificasse a necessidade do medicamento. Os grupos controle (GC) e experimental (GE) continham 35 alunos cada e foram submetidos respectivamente aos métodos de ensino tradicional e módulos instrucionais, conforme procedimento metodológico descrito por Nogueira (1995). A avaliação do aluno, em campo, foi realizada por enfermeira que desconhecia o tratamento específico a que cada aluno havia sido submetido e que, através de "Check-list" do procedimento para medicamento contido em ampola e frasco-ampola, seguira os passos realizados pelo aluno, indicando seu desempenho na coluna correspondente. Os resultados indicaram que o tempo gasto pelos sujeitos dos dois grupos foi praticamente o mesmo, ou seja, em média 9,4 minutos para o $\mathrm{GC}$ e 9,3 minutos para o $\mathrm{GE}$, quando realizaram o procedimento com ampola, e de 13,5 minutos (GC) e 12,4 minutos (GE), quando o fizeram com frasco-ampola. No geral, os alunos do Grupo Controle apresentaram, na situação real, desempenho regular $(42,9 \%)$, bom $(48,9 \%)$ e muito bom $(8,5)$, enquanto que os do Grupo Experimental classificaram-se em regular $(5,7 \%)$, bom $(60,0 \%)$ e muito bom (34,3\%). Estes resultados permitem evidenciar a importância do aluno se responsabilizar pela sua própria aprendizagem, levando-nos a utilizar novos métodos de ensino na enfermagem.
\end{abstract}

UNITERMOS: Ensino - Aprendizagem - Injetáveis via intramuscular

\footnotetext{
${ }^{1}$ Trabalho apresentado no $9^{\circ}$ SENPE - Vitória - Espírito Santo, julho de 1997.

2 Enfermeira, Professor Doutor - EERP-USP

${ }^{3}$ Enfermeira, Professor Titular - EERP-USP

${ }^{4}$ Enfermeira, Especialista em Laboratório - EERP-USP

${ }^{5}$ Enfermeira, Pró-Saúde - Departamento de Medicina Preventiva da São Francisco Clínicas - Ribeirão Preto-SP
} 


\begin{abstract}
The goal of the present study was to compare the performance of two groups of nursing undergraduate students, submetted to two different teaching methods, in their ability to performe the intramuscular injection on clients needing the procedure. Both, control (CG) and experimental group (EG) ( $n=35$ each) were submetted to traditional method and instrucional method respectively, according to the methodological procedure described by Nogueira (1995). The performance of the students was evaluated by a nurse who had no knowledge of the group each student belong. The nurse used the "check-list" in order to evaluate the performance of student. The results indicate that the time spent by the students of each group was approximatelly the same i - e 9,4 min for CG and 9,3 for EG. The students of the control group showed ordinary performance $(42,9 \%)$, good performance $(48,9 \%)$ and very good performance $(8,5 \%)$ whereas those of the EG showed ordinary performance $(5,7 \%)$, good performance $(60 \%)$ and very good performance $(34,3 \%)$. The present results shows that the students are responsible by their own learning, motivating us to use new teaching methods on nursing undergraduate course.
\end{abstract}

KEYWORDS: Teaching - Learning - Intramuscular injection.

Nossas inquietações motivaram-nos a desenvolver um estudo onde pudéssemos propor outro método de ensino que viesse contribuir para o melhor desenvolvimento da aprendizagem do aluno.

Por acreditarmos que 0 aluno deve ser participante ativo de sua aprendizagem e que, neste processo, deve-se considerar as diferenças individuais do mesmo, uma vez que cada um possui ritmo próprio, optamos por estratégia de ensino individualizada que pudesse ser viabilizada através de módulos instrucionais.

Considerando os fundamentos do ensino individualizado e as recomendações para sua organização, passamos a selecionar o conteúdo que iríamos oferecer por este método. Assim, foi escolhido o procedimento aplicação de injetáveis pela via intramuscular (IM).

A opção por este procedimento foi decorrente do nosso interesse em encontrar formas alternativas para o ensino do mesmo, além da exigência de habilidades que o desenvolvimento do procedimento requer para evitar a exposição do cliente a riscos.

Desta forma, o ensino de aplicação IM foi desenvolvido através de estudo experimental levado a efeito junto a alunos matriculados no curso de graduação em enfermagem, onde o grupo controle (GC) foi submetido ao método de ensino tradicional e o grupo experimental (GE) ao método individualizado, através de módulos instrucionais. No presente estudo, tivemos como objetivo avaliar o desempenho dos alunos do GC e GE durante sua primeira experiência realizando a aplicação de injetáveis via IM, em situação real. 


\section{METODOLOGIA}

Ao planejar o experimento, recorremos a um delineamento quase experimental, onde a classe foi dividida em dois grupos, sendo o GC e GE aleatórios.

Os 75 alunos matriculados na disciplina Cuidados Fundamentais de Enfermagem ao Homem Adulto, em 1994, foram distribuídos em GC e GE, através de amostragem aleatória; foram excluídos 5 alunos que já apresentavam experiência com o procedimento. Assim, a amostra ficou constituída por 70 alunos, dos quais os de números ímpares na lista de chamada foram colocados no GC e os de números pares, no GE. Cada um dos grupos ficou constituído por 35 alunos.

Aos alunos dos dois grupos, foram oferecias orientações básicas sobre o estudo e foi solicitada permissão para realização do mesmo, bem como a colaboração de todos, devido à necessidade de se testar outros métodos de ensino.

Após a divisão dos grupos, optamos por aplicar pré-teste visando garantir a homogeneidade, entre os alunos, quanto ao conhecimento básico sobre anatomia, especificamente em relação ao sistema muscular. Os resultados do pré-teste indicaram conhecimentos insuficientes, levando-nos a oferecer novamente este conteúdo aos dois grupos de alunos.

Posteriormente, cada grupo de aluno seguiu o programa de estudo previamente estabelecido, qual seja, o GC - método tradicional de ensino, (instrução oferecida por docente, em horário previamente determinada), e GE método de ensino através de módulos instrucionais (conforme fluxograma de estudo contendo 12 módulos, relacionados à aplicação de injetáveis via IM).

Após o estudo de cada módulo, o aluno respondia as questões para a aquisição de conhecimento, que 0 professor corrigia e indicava se 0 aluno poderia prosseguir nas demais atividades. Paralelamente, para a aquisição da habilidade motora, o aluno, após treinar sozinho o procedimento e sentir-se apto para realizá-lo, agendava horário com 0 monitor para devolução do procedimento. O monitor, de posse de "check-list" do módulo específico, observava o procedimento realizado pelo aluno e indicava os itens não realizados e/ou realizados incorretamente.

Quando todos os alunos, tanto do GC como GE, estavam na mesma fase de aquisição de conhecimento e de habilidade motora, realizou-se a avaliação da habilidade intelectual (situação teórica 1 e teórica 2) e habilidade motora (situação simulada, real 1 e real 2) comum aos dois grupos.

Para a avaliação do aluno, quer seja na habilidade intelectual ou motora, contratou-se enfermeira que desconhecia o tratamento específico a que cada aluno havia sido submetido. 
Para a determinação do nível de rendimento dos alunos, tanto na avaliação da habilidade intelectual como motora, utilizou-se a seguinte classificação: Péssimo - nenhum acerto; Ruim - 0,1 a 24,9\% de acertos; Regular - 25 a $49,9 \%$ de acertos; Bom - 50 a $74,9 \%$ de acertos; Muito Bom - 75 a $99,9 \%$ de acertos e Ótimo - $100 \%$ de acertos.

\section{RESULTADOS E DISCUSSÃO}

\section{Caracterização da amostra}

A amostra deste estudo foi composta de 70 sujeitos, alunos matriculados na disciplina Cuidados de Enfermagem ao Homem Adulto, da Escola de Enfermagem de Ribeirão Preto da Universidade de São Paulo, no ano de 1994, os quais foram distribuídos em grupo controle (GC) e grupo experimental (GE), com 35 alunos respectivamente.

Do total da amostra, $62(88,6 \%)$ estudantes são do sexo feminino e $8(14,4 \%)$ são do sexo masculino.

Em relação à idade, a faixa etária predominante foi de 18 a 21 anos com 49 (70\%) alunos, sendo que no grupo controle e experimental havia $60 \%$ e $80 \%$, respectivamente, de alunos nesta faixa etária.

Conforme já descrito na metodologia, os alunos que fizeram parte do grupo controle foram submetidos ao método de ensino referente à aplicação de injetáveis pela via intramuscular (IM), enquanto os sujeitos do grupo experimental desenvolveram estudo sobre o mesmo conteúdo, através de módulos instrucionais.

Desta forma, os resultados serão apresentados, analisados e discutidos mediante a comparação entre os dois grupos, especificamente em situação real.

\section{Situação real 1 - Tempo}

Esta situação caracteriza-se pelo primeiro momento em que o aluno realizou o procedimento em um cliente, e que constituiu também o primeiro procedimento invasivo de risco, realizado por ele nesta disciplina, em situação real, quando foi avaliado por uma enfermeira contratada especificamente para este fim, e acompanhado pela pesquisadora.

Esta etapa do estudo foi realizada em uma Unidade Básica de Saúde, onde posteriormente os alunos desenvolveriam as atividades teórico-práticas da disciplina sob orientação e supervisão de docente.

Para efeito deste estudo, considerou-se apenas a realização de aplicação de injetáveis pela via IM, em cliente adulto e que estivesse de posse de receita 
médica, onde os medicamentos receitados foram: Diclofenaco Sódico $75 \mathrm{mg}$, Penicilina G procaína 300.000 U com Penicilina G potássica $100.000 \mathrm{U}$, e Benzilpenicilina Benzatina 1.200.000U.

Assim, ao executarem o procedimento em situação real 1, os sujeitos dos grupos controle e experimental utilizaram o medicamento contido em ampola e frasco-ampola. Está especificado o tempo utilizado em cada fase do procedimento, onde os valores assinalados em negrito indicam a realização do procedimento com frasco-ampola. (Ver Tabela 1 em Anexo).

No grupo controle, os 27 sujeitos que realizaram o procedimento com medicamento contido em ampola utilizaram em média 9,4 minutos para executar o procedimento completo. $O$ tempo médio despendido para as fases de preparo do medicamento, preparo do paciente e aplicação do medicamento foi, respectivamente, 5,8; 2,4 e 1,2 minutos.

Os oito sujeitos do grupo controle que realizaram o procedimento utilizando medicamento contido em frasco-ampola, necessitaram de 13,5 minutos, em média, para realizar o procedimento completo. Para preparar o medicamento levaram em média 9,9 minutos e para o preparo do paciente e aplicação do medicamento precisaram em média de 2,3 e 1,4 minutos, respectivamente.

No grupo experimental, os 25 sujeitos que executaram o procedimento com medicamento contido em ampola levaram em média o tempo de 9,3 minutos para o procedimento como um todo; necessitaram de tempo médio de 5,6 minutos para a fase de preparo do medicamento, 2,6 minutos para o preparo do paciente e 1,1 minuto para aplicar o medicamento.

Os dez sujeitos restantes do grupo experimental que utilizaram medicamento contido em frasco-ampola, dispenderam em média 12,4 minutos para completar o procedimento e 9,3 minutos no preparo do medicamento, 2 minutos para o preparo do paciente e 1,1 minuto para a aplicação do medicamento.

De maneira geral, como nos anteriores, o comportamento dos grupos foi semelhante ou seja, foi dispendido mais tempo para a fase de preparo do medicamento, seguido da fase de preparo do paciente e por último, o que usou menos tempo, a aplicação do medicamento. Da mesma forma, nos dois grupos, - tempo médio utilizado é maior para o procedimento realizado com medicamento contido em frasco-ampola, quando comparado com aquele gasto com medicamento contido em ampola, sendo que os sujeitos do grupo controle necessitaram, no geral, de mais tempo do que os experimental.

Os dados da Tabela 1 permitem-nos inferir que, apesar desta situação ter se constituído da primeira experiência vivenciada pelo aluno, desenvolvendo um procedimento de risco invasivo junto ao cliente, parece não ter afetado a execução do procedimento, uma vez que o tempo utilizado nesta situação, pelos 
alunos que realizaram o procedimento com medicamento contido em ampola, difere pouco daquele dispendido na situação simulada. (Ver Anexo 1).

Considerando que o número de itens da fase de preparo do medicamento contido em frasco-ampola é de 28 itens e a daquele contido em ampola é de 16 itens, justifica-se a utilização de maior tempo para realizar esta fase, pelos dois grupos que utilizaram frasco-ampola.

Nas fases de preparo do paciente e aplicação do medicamento, os alunos de ambos os grupos apresentaram o mesmo comportamento em relação ao tempo, quer seja utilizando medicamento em frasco-ampola ou ampola, quando comparados à situação simulada. Este dado indica que o aluno, neste momento do curso, ainda não é capaz de discernir sobre as complicações que envolvem a aplicação de medicamentos, uma vez que entre preparar um "paciente" para receber $0,5 \mathrm{ml}$ de SF $0,9 \%$ e outro para receber $4 \mathrm{ml}$ de Benzilpenicilina Benzatina 1.200.000U, acaba investindo mais tempo no primeiro caso, ou seja, naquele em que as complicações decorrentes da sua aplicação podem ser menos graves em termos do "medicamento"em si.

Este resultado é confirmado, mais uma vez, quando se compara a média de tempo utilizado pelos sujeitos dos dois grupos, quando na situação real, ao efetuarem a aplicação de Diclofenaco Sódico $75 \mathrm{mg}$, Benzilpenicilina Benzatina 1.200.000U e Penicilina G Procaína 300.000 U com Penicilina G Benzatina $100.000 \mathrm{U}$ pela via IM; dispenderam mais tempo na fase de preparo do paciente para a aplicação do Diclofenaco Sódico $75 \mathrm{mg}$.

Ocorrências desta ordem reforçam nossa compreensão de que o aluno deve ser acompanhado na realização de procedimentos junto ao cliente, uma vez que diante de situações novas ele parece impossibilitado de pensar e, neste momento, alguém deve pensar por ele, no caso o professor, para impedir que exponha o cliente a riscos.

Deve-se considerar ainda, que a utilização de tempo maior para a fase de preparo do paciente não esteve relacionada, na maioria das vezes, a um maior interesse do aluno para a interação com o cliente, e sim à falta de habilidade em delimitar a região escolhida para a aplicação do medicamento e também à indecisão no momento de introduzir a agulha. Neste momento, o aluno parece estar mais preocupado em realizar o procedimento, o que impede de ver o cliente, porque "há algo que solicita mais sua atenção, no caso o procedimento" (Angelo, 1989).

Esta percepção é decorrente de nossas observações informais enquanto o aluno estava realizando o procedimento e podem ser confirmadas pelo tempo escasso que alguns alunos, tanto do grupo controle como do experimental, investiram nesta fase, apenas um minuto. 
Sabe-se, no entanto, que este tempo de um minuto pode ser insuficiente, considerando as orientações a serem fornecidas ao cliente, uma vez que os alunos deveriam convencê-lo sobre a possibilidade da utilização de outras regiões para a aplicação de medicamentos pela via IM, que não apenas a região deltóidea, preferida pela maioria das pessoas.

Shallowhorn (1954), ao tecer considerações sobre a administração de medicamentos por via intramuscular, declara que uma das responsabilidades primárias do enfermeiro, ao realizar este procedimento, está no preparo físico do paciente.

Miyadahira (1990) encontrou que o grupo de alunos que executou a técnica de IM em pacientes, no hospital ou posto de saúde, dispendeu maior tempo que o grupo que executou o procedimento em laboratório, e justifica que o maior tempo utilizado por estes alunos estava associado aos cuidados e orientações específicas fornecidas ao paciente.

A mesma autora considera ainda que, a partir do momento em que "ocorre a incorporação de um conhecimento previamente configurado, o tempo gasto não deve influir de maneira significativa na experiência anterior, pois os passos a serem seguidos serão sempre os mesmos, e o tempo gasto dependerá muito mais da atenção que o paciente/cliente requisita do que a técnica em si".

\section{Situação real 1 - Desempenho}

Considerando que nesta situação os alunos necessitam realizar o procedimento ora com medicamento contido em ampola ora em frasco-ampola, para efeito de comparação do desempenho dos sujeitos em ambos os grupos, optou-se por considerar apenas os itens em comum com a situação simulada, onde os sujeitos executaram o procedimento com medicamento contido em ampola, e que portanto compreendia a execução de 34 itens.

Os dados referentes ao desempenho dos sujeitos dos grupos controle e experimental, ao executarem o procedimento em situação real 1, podem ser observados na Tabela 2 em Anexo 1.

Os sujeitos do grupo controle acertaram em média 18,1 itens, ou seja, $53,4 \%$ dos itens do procedimento na situação real 1. Obtiveram melhor desempenho na fase de preparo do medicamento, onde acertaram em média $9,7 \%$ itens, ou $60,7 \%$ dos itens desta fase. Nas outras duas fases não acertaram nem metade do número de itens necessários, alcançando média de acerto de 6,2 itens (47,7\% dos itens) da fase de aplicação do medicamento e de 2,2 itens $(44,6 \%$ dos itens) da fase de preparo do paciente.

Para os sujeitos do grupo experimental, a média de acerto foi de 24,1 itens, o que corresponde a $70,8 \%$ dos itens do procedimento. 
O melhor desempenho, entre as fases, também ocorreu na de preparo do medicamento, onde houve acerto médio de 12,8 itens ( $80 \%$ do total de itens da fase). Durante a aplicação do medicamento, acertaram em média 8,5 itens (65,1\% dos itens da fase) e, ao executarem o preparo do paciente, o acerto médio foi de 2,8 itens ( $56,6 \%$ dos itens da fase).

$\mathrm{Na}$ situação real 1 , os sujeitos de ambos os grupos tiveram o mesmo comportamento em relação às fases do procedimento, ou seja, ambos tiveram mais dificuldades na fase de preparo do paciente e menos, na de preparo do medicamento.

Diante dos achados e comparando-se a performance entre os sujeitos dos dois grupos, pode-se verificar que também nesta situação o grupo experimental apresentou melhor desempenho, com índice de acerto maior do que o do grupo controle.

Através da aplicação da Prova $U$ de Mann-Whitney podemos comprovar que a diferença do número de acertos é estatisticamente significante ao nível de $p<0,00001(z=4,69)$, confirmando melhor desempenho dos sujeitos do grupo experimental sobre os do grupo controle.

Da comparação da porcentagem de acertos entre os dois grupos para as três fases do procedimento, verifica-se que, nesta situação, houve uma inversão em todas as fases, quando comparadas à situação simulada.

Como já dito anteriormente, essa situação já é esperada, uma vez que a vivência deste primeiro contato do aluno com o paciente reveste-se de muita ansiedade e estresse como já apontado por autores como Grigby e Smith (1977); French (1980); Infante (1981); Friedlander (1984) e Angelo (1989).

O fato dos alunos terem errado mais na situação real 1 a fase de preparo do paciente e tê-la acertado mais na situação simulada pode ser explicado considerando-se dois aspectos, a seguir apresentados.

O primeiro pode estar associado ao reduzido número de erros apresentados nesta fase na situação simulada, impedindo-nos de visualizá-la como problemática, e, portanto, não sentindo necessidade de reforçar o desenvolvimento da mesma.

O segundo aspecto está vinculado ao estresse gerado pela primeira experiência de execução deste procedimento num cliente, o que dificultou 0 estabelecimento de interação com o mesmo. Alguns alunos permaneciam diante do cliente quase que imóveis, com o medicamento preparado, como se esperassem sua reação frente à visão da seringa. Iniciavam qualquer diálogo somente quando o cliente oferecia um dos braços para que fosse aplicado o medicamento. Diante desta reação, muitos alunos conseguiam iniciar orientação sobre as desvantagens da utilização da região deltóidea e sugerir outras regiões 
mais adequadas, considerando o volume do medicamento, a massa muscular do cliente e o rodizio de locais de aplicação.

O estudo de Horr et al (1978) mostrou que $50 \%$ dos enfermeiros referiam orientar os pacientes sobre o local de aplicação de IM; entre os auxiliares de enfermagem, este resultado foi de $63 \%$. Ao confrontarem estes resultados com as informações fornecidas pelos pacientes, foi possível identificar que apenas $3 \%$ daqueles que tinham condições de serem entrevistados indicaram que haviam recebido informações.

Ao estudarem $o$ efeito da ansiedade sobre a performance da habilidade motora entre alunos de enfermagem, Embury \& Thurston (1976) afirmam que os alunos experimentam grande ansiedade ao desenvolverem procedimentos de enfermagem, especificamente a administração de injeções.

Quanto às primeiras experiências práticas do aluno, com o desenvolvimento de procedimento para um cliente, Angelo (1989) coloca que "neste início é difícil para a aluna fazer as duas coisas, explicar ao paciente e prestar atenção à técnica. Assim, o fato de ter que realizar o procedimento pela primeira vez e não se sentir ainda segura, faz com que só consiga enxergar o procedimento".

Por outro lado, acredita-se que nesta situação, a inversão de prioridade de acertos para a fase de aplicação do medicamento, quando comparada com a situação simulada, esteja relacionada à necessidade de orientação percebida, pelo professor, sobre a forma de execução desta fase, quando os alunos realizaram o procedimento na situação anterior.

Sobre este aspecto, Bloom (1981) considera que, se as dificuldades na primeira tarefa não forem corrigidas, serão provavelmente acrescidas de maiores dificuldades também nas tarefas subseqüentes.

Neste caso, parece que o "feedback" fornecido ao aluno, por ocasião da realização do procedimento em situação simulada sobre seus erros e acertos, contribui para que melhorasse o seu desempenho ao realizar o procedimento na situação real; especificamente nas fases de preparo e de aplicação do medicamento.

Ao considerar a execução global da técnica de IM entre os alunos matriculados no $3^{\circ}, 4^{\circ}$ e $8^{\circ}$ semestres do curso de enfermagem, Miyadahira (1990) encontrou que $50,36 \%$ dos estudantes de sua amostra citaram o item referente à necessidade de explicar ao paciente, enquanto que apenas $8,63 \%$ deles fizeram referência sobre a posição do paciente, quando solicitados a descreverem o procedimento de IM.

Comparando os nossos achados com os de Miyadahira (1990), fica evidenciado que os alunos não atribuem muita importância à necessidade de explicar ao cliente o que estão ou vão realizar. Estes dados causam-nos 
estranheza uma vez que é comum encontrar, entre os alunos, a valorização desta conduta quando a descrevem em situação teórica, o que também pode ser percebido nas críticas que normalmente fazem quando observam algum elemento da equipe executando qualquer procedimento sem privilegiar a etapa relacionada à informação fornecida ao cliente, sobre sua ação.

$\mathrm{Na}$ Tabela 3 estão descritos os dados referentes ao nível de desempenho dos sujeitos dos grupos controle e experimental ao realizarem o procedimento nas situações simuladas, real 1 e real 2 .

TABELA 3 - NÚMERO E PORCENTAGEM DE SUJEITOS DOS GRUPOS CONTROLE E EXPERIMENTAL DE ACORDO COM O NIVEL DE DESEMPENHO AO REALIZAREM O PROCEDIMENTO EM SITUAÇÃO SIMULADA, REAL 1 E REAL 2.

\begin{tabular}{|c|c|c|c|c|c|c|c|c|c|c|c|c|}
\hline \multirow{4}{*}{ Desempenho } & \multicolumn{6}{|c|}{ GRUPO CONTROLE } & \multicolumn{6}{|c|}{ GRUPO EXPERIMENTAL } \\
\hline & \multicolumn{6}{|c|}{ SITUAÇÃO } & \multicolumn{6}{|c|}{ SITUAÇÃO } \\
\hline & \multicolumn{2}{|c|}{ SIMULADA } & \multicolumn{2}{|c|}{ REAL 1} & \multicolumn{2}{|c|}{ REAL 2} & \multicolumn{2}{|c|}{ SIMULADA } & \multicolumn{2}{|c|}{ REAL 1} & \multicolumn{2}{|c|}{ REAL 2} \\
\hline & No & $\%$ & № & $\%$ & $\mathrm{~N}^{0}$ & $\%$ & $\mathrm{~N}^{\circ}$ & $\%$ & $\mathrm{~N}^{\circ}$ & $\%$ & $\mathrm{~N}^{0}$ & $\%$ \\
\hline Péssimo & . & - & - & - & - & - & - & - & - & - & $=$ & - \\
\hline Ruim & - & - & - & - & - & - & - & - & - & - & - & - \\
\hline Regular & - & - & 15 & 42,9 & 10 & 28,6 & - & - & 2 & 5,7 & 1 & 2,9 \\
\hline Bom & 21 & 60 & 17 & 48,6 & 21 & 60,0 & 2 & 5,7 & 21 & 60,0 & 14 & 40,0 \\
\hline Muito Bom & 14 & 40 & 3 & 8,5 & 4 & 11,4 & 32 & 91,4 & 12 & 34,3 & 20 & 57,1 \\
\hline Ótimo & - & - & - & - & - & - & 1 & 2,9 & - & - & - & - \\
\hline TOTAL & 35 & 100 & 35 & 100,0 & 35 & 100,0 & 35 & 100,0 & 35 & 100,0 & 35 & 100,0 \\
\hline
\end{tabular}

Assim, ao analisarmos o desempenho dos dois grupos na situação simulada, pode-se verificar que, no grupo controle, $21(60 \%)$ sujeitos foram classificados no nível bom e 14 (40\%) no nível muito bom desempenho, enquanto que, para o grupo experimental, $32(91,4 \%)$ sujeitos enquadram-se no nível de muito bom desempenho, $2(5,7 \%)$ no nível bom e $1(2,9 \%)$ no nível ótimo desempenho.

Para a situação real 1 , o grupo controle apresentou $17(48,6 \%)$ sujeitos classificados no nivel de bom desempenho, $15(42,9 \%)$ no nível regular e 3 $(8,5 \%)$ no de muito bom desempenho. Por outro lado, no grupo experimental, 21 $(60 \%)$ sujeitos tiveram sua classificação no nível bom, $12(34,3 \%)$ no nível muito bom e $2(5,7 \%)$ sujeitos no nivel regular.

$\mathrm{Na}$ situação real 2, constata-se mais uma vez a superioridade do desempenho dos sujeitos do grupo experimental, onde $20(57,1 \%)$ sujeitos 
enquadram-se no nível muito bom, $14(40 \%)$ no nível bom e $1(2,9 \%)$ no nível regular; enquanto no grupo controle, 21 (60\%) sujeitos ficaram no nivel bom, 10 $(28,6 \%)$ no regular e $4(11,5 \%)$ no nível de muito bom desempenho.

Ao considerarmos o desempenho dos alunos dos dois grupos nas três situações, podemos dizer que o grupo controle parece ter sofrido maior impacto quando colocado para realizar o procedimento na situação real, uma vez que nela foi grande o número de alunos classificados no nível regular $(42,9 \%)$, enquanto que a maioria dos alunos do grupo experimental obteve classificação no nivel de bom desempenho $(60 \%)$.

Após as comparações feitas entre o desempenho dos grupos para as três situações, optamos agora por confrontar o desempenho dos sujeitos do mesmo frente às três situações observadas. Assim, utilizamos o Teste de Wilcoxon para comparar mais de dois tratamentos (Two-Way).

Desta forma, encontramos que tanto para o grupo controle como para o experimental, as diferenças entre a somatória de postos das três situações, como demostradas a seguir, só foram estatisticamente significativa $(\propto=0,01)$ na situação simulada $(A)$, quando comparada às situações real $1(B)$ e real $2(C)$, as quais estão assinaladas com asterisco. Portanto, as situações reais 1 e 2 , quando comparadas entre si, não foram significativas em ambos os grupos.

Somatória dos postos obtidos pelos sujeitos dos grupos controle e experimental nas situações simulada (A), real 1 (B) e real 2 (C) e as diferenças quando comparadas entre si pelo teste de Wilcoxon para mais de dois tratamentos (Two-way)

\begin{tabular}{c|c|c|c}
\hline \multirow{2}{*}{ Situaçōes } & \multicolumn{3}{|c}{ grupo CONTROLE } \\
\cline { 2 - 4 } & A & C & B \\
\hline & 94,5 & 61,5 & 54,0 \\
A 94,5 & - & - & - \\
C 61,5 & $33,0^{*}$ & - & - \\
B 54,0 & $40,5^{*}$ & 7,5 & - \\
\hline
\end{tabular}

\begin{tabular}{|c|c|c|c|}
\hline \multirow[b]{2}{*}{ Situação } & \multicolumn{3}{|c|}{ grupo EXPERIMENTAL } \\
\hline & A & C & B \\
\hline & 99,0 & 59,0 & 52,0 \\
\hline A 99,0 & - & - & - \\
\hline C 59,0 & $40,0^{*}$ & - & - \\
\hline B 52,0 & $47,0^{*}$ & 7,0 & - \\
\hline
\end{tabular}

* Diferenças significantes $(\propto=0,01)$

Com base nestes dados, concluímos que o desempenho dos sujeitos de ambos os grupos foi significativamente melhor na situação simulada $(A)$ do que nas situações real 2 (C) e real 1 (B). Entretanto, quando comparamos as duas situações reais (B e C) o desempenho foi o mesmo, ou seja, a diferença de melhor desempenho na situação real 2 (C) em relação à real 1 (B) não foi significativa.

Estes achados permitem-nos concluir que, quando executou o procedimento na situação real 2, apesar de ter tido mais oportunidades de realizá-lo em maior número de vezes do que em situação real 1 , isto não assegurou que fosse 
suficiente para ocorrer maior retenção da habilidade motora e conhecimento sobre o procedimento.

Desta forma, nossos achados encontram reforço no estudo de Miyadahira (1990) quando diz que o "conhecimento e a habilidade psicomotora da técnica de IM só estavam consolidados entre os alunos, a partir do $4^{\circ}$ semestre".

$O$ fato de apresentarem melhor desempenho, estatisticamente siginificante, em situação simulada quando comparada à situação real, mostra-nos que a utilização apenas da situação simulada não se constituiu em alternativa suficiente para o desenvolvimento do ensino, e sim como recurso adicional de instrução.

Os dados arranjados desta forma permitem identificar, mais uma vez, que 0 desempenho dos alunos do grupo experimental foi superior àquele do grupo controle. Ao analisarmos especificamente o desempenho dos alunos dos dois grupos, na situação real 2, podemos identificar que o rendimento dos alunos do grupo controle se distribui segundo uma curva normal.

Neste aspecto, e considerando as relações entre as variáveis aptidão, instrução e rendimento a que Penteado(1980) faz referência e que são estabelecidas por Carroll, pode-se afirmar que os resultados comprovam a concepção de ensino tradicional para o grupo controle. De acordo com o referido autor, se a aptidão entre os alunos se distribui segundo uma curva normal, é de se esperar que o rendimento entre eles também obedeça a mesma distribuição, uma vez que "algumas pessoas são extremamente dotadas, outras bastante limitadas e um bom número delas medianamente dotadas". Assim, ao estabelecer conexão causal entre aptidão e aproveitamento, Penteado(1980) considera que "altos índices de aproveitamento somente podem ser alcançados por alunos bem dotados".

Por outro lado considera que, se a aptidão for distribuída segundo uma curva normal e aos alunos for oferecida instrução de ótima qualidade, o rendimento poderá ser muito melhor.

Neste estudo, e considerando especificamente o melhor desempenho do grupo experimental, acredita-se que tal fato esteja relacionado ao maior tempo que se dedicaram ao estudo e também ao acompanhamento individual que o aluno teve por parte do professor ou do monitor, durante a fase de avaliação formativa.

Este achado vai ao encontro com as variáveis que Carroll considera que atuam na aprendizagem do aluno, e são de responsabilidade do professor: a qualidade da instrução e o tempo concedido ao aluno para o estudo. Sobre a qualidade da instrução, é importante ressaltar que o conteúdo selecionado para construção dos módulos instrucionais foi o mesmo que a pesquisadora utilizou para ministrar aula teórica e prática aos alunos do grupo controle. Desta forma, acreditamos que o melhor desempenho do grupo experimental, sem dúvida, está 
relacionado à possibilidade do acompanhamento individual do aluno por ocasião dos treinos em laboratório, para o desenvolvimento de habilidades psicomotoras, e à forma de apresentação do conteúdo, que atende ao ritmo de aprendizagem de cada um.

Por outro lado, o estudo através de módulos instrucionais demonstra ter sido aprovado, tendo em vista que não mediram esforços para cumprirem as atividades propostas, cujo empenho pode ser verificado pela presença no laboratório em horários de almoço, final da tarde e finais de semana.

Este empenho, no entanto, possibilitou economia de tempo dos alunos do grupo experimental para o estudo referente ao conteúdo específico, o que indica a perseverança que Carroll considera ser uma variável relacionada às características do aluno para a aprendizagem. Acreditamos que a manutenção da presença do aluno para completar o estudo dos módulos esteve relacionada ao interesse dos mesmos para a aprendizagem deste conteúdo específico.

Deve-se considerar ainda que outros fatores que contribuíram para a perseverança do aluno foram: a) o oferecimento do conteúdo em pequenas partes e de modo gradativo e b) a avaliação formativa ter ocorrido no final do estudo de cada módulo, o que se constituiu em esforço positivo, uma vez que permitiu ao aluno conhecer seu próprio desempenho, receber ensino, quando o monitor e professor julgassem necessários e através de sua própria necessidade.

Ao considerarmos a variável relacionada à capacidade do aluno para compreender a instrução, acreditamos que neste estudo os alunos do grupo experimental podem ter sido privilegiados, pois, à medida que sentiam dificuldades sobre a tarefa de aprendizagem, procuravam esclarecimentos. Outra alternativa que contribuiu para destacar esta variável foi a opção do aluno para a escolha de diferentes tarefas de aprendizagem.

\section{Situação real 1 - Procedimento com medicamento contido em ampola}

O número e as médias de erros e acertos dos sujeitos de ambos os grupos, durante as fases do procedimento realizado em situação real 1, estão na Tabela 4, Anexo 3.

Em situação real 1, o grupo controle teve uma média geral de acerto de 18,5 itens, o equivalente a $54,6 \%$ dos itens do procedimento. Considerando as fases do procedimento, acertaram em média 10 itens $(62,3 \%)$ da fase de preparo do medicamento, 2,4 itens $(48,1 \%)$ da fase de preparo do paciente e 6,2 itens $(47,6 \%)$ da fase de aplicação do medicamento.

Quanto ao grupo experimental, os sujeitos acertaram em média 25,4 itens $(74,8 \%)$ do procedimento com ampola. Durante as fases, o acerto médio foi de 13,2 itens $(82,5 \%)$ da fase de preparo do medicamento, 3 itens $(60,8 \%)$ da fase 
de preparo do paciente e 9,2 itens $(70,8 \%)$ da fase de aplicação do medicamento.

Através destes dados é possível verificar que, ao executarem o procedimento em situação real 1 , os sujeitos do grupo experimental apresentaram melhor desempenho que os do grupo controle. Para constatar se esta superioridade é significativa aplicamos a Prova $U$ de Mann-Whitney comparando o número de acerto dos sujeitos de ambos os grupos.

Com base no resultado desta Prova, concluímos que a diferença é altamente significativa ao nivel de $p<0,00003(z=-4,587)$, ou seja, o desempenho dos sujeitos do grupo experimental foi significativamente maior do que 0 do outro grupo.

\section{Situação real 1 - Procedimento com medicamento contido em frasco- ampola}

Os dados referentes ao número e média de erro e acerto dos sujeitos dos grupos controle e experimental durante as fases do procedimento com medicamento contido em frasco-ampola, em situação real 1, estão na Tabela 5, Anexo 4.

Para o grupo controle, em situação real 1 , as médias de acerto foram de 21 itens que correspondem a $45,7 \%$ do total de itens do procedimento e de 13,1 itens $(46,9 \%)$ da fase de preparo do medicamento, 1,6 itens $(32,5 \%)$ da fase de preparo do paciente e 6,3 itens $(48,1 \%)$ da fase de aplicação do medicamento.

No grupo experimental, os valores médios de acerto foram de 27 itens $(58,7 \%)$ do procedimento com frasco-ampola, 18,1 itens $(64,6 \%)$ da fase de preparo do medicamento, 2,3 itens $(46 \%)$ da fase de preparo do paciente e 6,6 itens $(50,8 \%)$ da fase de aplicação do medicamento.

O desempenho dos sujeitos do grupo experimental, ao realizarem o procedimento com frasco-ampola, é melhor do que aquele desenvolvido pelos do outro grupo. Para comprovar se esta diferença é significativa, aplicamos a Prova $U$ de Mann-Whitney, que mostrou mais uma vez a superioridade do grupo experimental quando comparado com o grupo controle ao nível de significância $\propto=0,05(U=18)$.

Procurando comparar o desempenho dos grupos de outra forma, enquadramos os sujeitos em categoria de nível de desempenho conforme mostra a Tabela 6 , onde estão os números e porcentagens de sujeitos dos grupos controle e experimental, de acordo com o nivel de desempenho ao executarem o procedimento com frasco-ampola, em situações real 1 e real 2. 
TABELA 6 - NÚMERO E PORCENTAGEM DE SUJEITOS DOS GRUPOS CONTROLE E EXPERIMENTAL DE ACORDO COM O NIVELL DE DESEMPENHO AO REALIZAREM O PROCEDIMENTO COM FRASCO-AMPOLA (46 ITENS) EM SITUAÇÄO REAL 1 E REAL 2.

\begin{tabular}{|c|c|c|c|c|c|c|c|c|}
\hline \multirow{3}{*}{$\begin{array}{c}\text { NIVEL } \\
\text { DE } \\
\text { DESEMPENHO }\end{array}$} & \multicolumn{4}{|c|}{$\frac{\text { GRUPO CONTROLE }}{\text { SITUACAAO }}$} & \multicolumn{4}{|c|}{$\begin{array}{l}\text { GRUPO EXPERIMENTAL } \\
\text { SITUACAO }\end{array}$} \\
\hline & \multicolumn{2}{|c|}{ REAL 1} & \multicolumn{2}{|c|}{ REAL 2} & \multicolumn{2}{|c|}{ REAL 1} & \multicolumn{2}{|c|}{ REAL 2} \\
\hline & $\mathrm{N}^{\circ}$ & $\%$ & $\mathrm{~N}^{0}$ & $\%$ & $\mathrm{~N}^{\circ}$ & $\%$ & $\mathrm{~N}^{0}$ & $\%$ \\
\hline Péssimo & 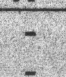 & - & 1 & 77 & - & 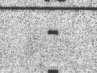 & - & 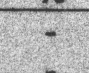 \\
\hline $\begin{array}{l}\text { Ruim } \\
\text { Regular }\end{array}$ & 5 & 62,5 & 7 & 53.8 & 3 & 30.0 & 1 & 8.3 \\
\hline Bom & 3 & 37,5 & 4 & 30,8 & 7 & 70,0 & 4 & 33,3 \\
\hline Muito Bom & - & - & 1 & 7,7 & - & . & 7 & 58,4 \\
\hline Ótimo & - & $=$ & - & & - & - & - & \\
\hline Total & 8 & 100,0 & 13 & 100,0 & 10 & 100,0 & 12 & 100,0 \\
\hline
\end{tabular}

Em situação real 1 para executar o procedimento com frasco-ampola, o grupo controle teve 5 sujeitos $(62,5 \%)$ classificados no nível de desempenho regular, os demais, 3 sujeitos $(37,5 \%)$ no nível bom; no grupo experimental, 7 sujeitos $(70,0 \%)$ foram classificados no nível de desempenho bom e $3(30,0 \%)$ em nivel regular.

Em relação ao desempenho na situação real 2, no grupo controle, 7 (53,8\%) sujeitos estão no nível de desempenho regular, $4(30,8 \%)$ no nível bom e igualmente um sujeito $(7,7 \%)$ nos níveis muito bom e ruim. Por outro lado, 7 sujeitos $(58,4 \%)$ do grupo experimental tiveram desempenho muito bom e os demais ficaram entre níveis bom 4 sujeitos $(33,35 \%)$ e regular um sujeito $(8,3 \%)$.

Estes resultados permitem confirmar que, ao realizar o procedimento com frasco-ampola, o aluno apresenta nivel de desempenho inferior, pois, entre os alunos do grupo controle que realizaram o procedimento com medicamento nesta forma de apresentação, há um maior número de alunos classificados no nivel de desempenho regular, tanto para a situação real 1 quanto para a real 2. Para o grupo experimental, no entanto, pode-se identificar que a execução do procedimento com ampola foi responsável pela classificação de alunos no nível de desempenho regular nas duas situações.

Fica evidenciado, dessa forma, que os alunos apresentam maior dificuldade para realizar o procedimento com o medicamento contido em frasco-ampola, o que nos indica a necessidade de propiciar maior oportunidade de treino nesta modalidade.

\section{CONCLUSÕES}

A superioridade de desempenho dos alunos do grupo experimental, comparada ao do grupo controle, permite-nos evidenciar a importância do aluno se responsabilizar pela sua própria aprendizagem, levando-nos a utilizar novos métodos de ensino na enfermagem. 


\section{ANEXO 1}

Tabela 1 - Tempo utilizado, em minutos, pelos sujeitos dos grupos controle e experimental ao executarem o procedimento em situação real 1 , e tempo médio utilizado para realizar o procedimento com medicamento contido em ampola (A) e em frasco-ampola (FA), de acordo com as fases de preparo do medicamento (PM), preparo do paciente (PP) e aplicação do medicamento (AM).

\begin{tabular}{|c|c|c|c|c|c|c|c|c|}
\hline \multirow{2}{*}{ SUJEITOS } & \multicolumn{4}{|c|}{ GRUPO CONTROLE } & \multicolumn{4}{|c|}{ GRUPO EXPERIMENTAL } \\
\hline & PM & PP & $A M$ & TOTAL & PM & PP & AM & TOTAL \\
\hline 1 & 8 & 2 & 1 & 11 & 3 & 3 & 2 & 8 \\
\hline 2 & 6 & 5 & 1 & 12 & 5 & 4 & 1 & 10 \\
\hline 3 & 5 & 8 & 1 & 14 & 6 & 1 & 1 & 8 \\
\hline 4 & 4 & 2 & 2 & 8 & 4 & 2 & 1 & 7 \\
\hline 5 & 10 & 1 & 1 & 12 & 6 & 1 & 1 & 8 \\
\hline 6 & 9 & 3 & 1 & 13 & 9 & 1 & 1 & 11 \\
\hline 7 & 5 & 2 & 1 & 8 & 11 & 2 & 1 & 14 \\
\hline 8 & 6 & 2 & 1 & 9 & 4 & 2 & 1 & 7 \\
\hline 9 & 6 & 3 & 2 & 11 & 8 & 1 & 1 & 10 \\
\hline 10 & 4 & 3 & 2 & 9 & 7 & 2 & 1 & 10 \\
\hline 11 & 5 & 2 & 1 & 8 & 6 & 3 & 1 & 10 \\
\hline 12 & 4 & 1 & 1 & 6 & 7 & 1 & 1 & 9 \\
\hline 13 & 6 & 3 & 1 & 10 & 6 & 3 & 1 & 10 \\
\hline 14 & 10 & 3 & 1 & 14 & 6 & 3 & 1 & 10 \\
\hline 15 & 7 & 1 & 1 & 9 & 8 & 3 & 1 & 12 \\
\hline 16 & 5 & 3 & 1 & 9 & 6 & 2 & 1 & 9 \\
\hline 17 & 9 & 1 & 2 & 12 & 9 & 3 & 1 & 13 \\
\hline 18 & 6 & 2 & 1 & 9 & 7 & 3 & 1 & 11 \\
\hline 19 & 5 & 1 & 1 & 7 & 5 & 3 & 1 & 9 \\
\hline 20 & 12 & 4 & 1 & 17 & 6 & 2 & 1 & 9 \\
\hline 21 & 6 & 2 & 1 & 9 & 6 & 5 & 1 & 12 \\
\hline 22 & 6 & 2 & 1 & 9 & 9 & 1 & 1 & 11 \\
\hline 23 & 9 & 3 & 3 & 15 & 4 & 3 & 1 & 8 \\
\hline 24 & 6 & 1 & 1 & 8 & 5 & 1 & 1 & 7 \\
\hline 25 & 5 & 2 & 2 & 9 & 9 & 2 & 1 & 12 \\
\hline 26 & 6 & 3 & 1 & 10 & 10 & 1 & 2 & 13 \\
\hline 27 & 8 & 4 & 1 & 13 & 12 & 1 & 1 & 14 \\
\hline 28 & 5 & 1 & 1 & 7 & 6 & 4 & 1 & 11 \\
\hline 29 & 8 & 1 & 2 & 11 & 5 & 3 & 1 & 9 \\
\hline 30 & 6 & 3 & 1 & 10 & 5 & 3 & 1 & 9 \\
\hline 31 & 6 & 2 & 1 & 9 & 5 & 3 & 1 & 9 \\
\hline 32 & 6 & 2 & 1 & 9 & 12 & 3 & 1 & 16 \\
\hline 33 & 5 & 1 & 1 & 7 & 8 & 6 & 2 & 16 \\
\hline 34 & 7 & 2 & 2 & 11 & 5 & 1 & 2 & 8 \\
\hline 35 & 15 & 1 & 1 & 17 & 3 & 3 & 1 & 7 \\
\hline TOTAL (A) & 157 & 64 & 33 & 254 & 140 & 65 & 28 & 233 \\
\hline TOTAL (FA) & 79 & 18 & 11 & 108 & 93 & 20 & 11 & 124 \\
\hline MÉDIA (A) & 5,8 & 2,4 & 1,2 & 9,4 & 5,6 & 2,6 & 1,1 & 9,3 \\
\hline MÉDIA (FA) & 9,9 & 2,3 & 1,4 & 13,5 & 9,3 & 2,0 & 1,1 & 12,4 \\
\hline
\end{tabular}

NOTA: Os valores assinalados em negrito correspondem à execução do procedimento com frascoampola (46 itens). Os demais, à execução com ampola (34 itens). 


\section{ANEXO 2}

Tabela 2 - Número, média e porcentagem de erro (E) e acerto (A) dos itens que compõem as fases de preparo do medicamento (PM) contido em ampola, preparo do paciente (PP) e aplicação do medicamento (AM) para cada sujeito dos grupos controle e experimental ao realizar o procedimento em situação real 1

\begin{tabular}{|c|c|c|c|c|c|c|c|c|c|c|c|c|c|c|c|c|}
\hline \multirow{4}{*}{ SUIEITOS } & \multicolumn{8}{|c|}{ GRUPO CONTROLE } & \multicolumn{8}{|c|}{ GRUPO EXPERIMENTAL } \\
\hline & \multicolumn{8}{|c|}{ FASE } & \multicolumn{8}{|c|}{ FASE } \\
\hline & \multicolumn{2}{|c|}{ PM } & \multicolumn{2}{|c|}{$P P$} & \multicolumn{2}{|l|}{ AM } & \multicolumn{2}{|c|}{ TOTAL } & \multicolumn{2}{|c|}{ PM } & \multicolumn{2}{|c|}{$\mathrm{PP}$} & \multicolumn{2}{|c|}{ AM } & \multicolumn{2}{|c|}{ TOTAL } \\
\hline & $E$ & A & $E$ & A & $E$ & A & $E$ & $A$ & $E$ & A & $E$ & A & $E$ & $A$ & $E$ & A \\
\hline 1 & 6 & 10 & 4 & 1 & 9 & 4 & 19 & 15 & 2 & 14 & 2 & 3 & 5 & 8 & 9 & 25 \\
\hline 2 & 13 & 3 & 4 & 1 & 7 & 6 & 24 & 10 & 2 & 14 & 0 & 5 & 5 & 8 & 7 & 27 \\
\hline 3 & 8 & 8 & 1 & 4 & 3 & 10 & 12 & 22 & 2 & 14 & 2 & 3 & 5 & 8 & 9 & 25 \\
\hline 4 & 1 & 15 & 2 & 3 & 8 & 5 & 11 & 23 & 4 & 12 & 3 & 2 & 2 & 11 & 9 & 25 \\
\hline 5 & 6 & 10 & 4 & 1 & 8 & 5 & 18 & 16 & 7 & 9 & 2 & 3 & 2 & 11 & 11 & 23 \\
\hline 6 & 9 & 7 & 5 & 0 & 8 & 5 & 22 & 12 & 5 & 11 & 1 & 4 & 2 & 11 & 8 & 26 \\
\hline 7 & 8 & 8 & 4 & 1 & 9 & 4 & 21 & 13 & 5 & 11 & 5 & 0 & 8 & 5 & 18 & 16 \\
\hline 8 & 3 & 13 & 4 & 1 & 13 & 0 & २० & $\cdot 14$ & 3 & 13 & 2 & 3 & 5 & 8 & 10 & 24 \\
\hline 9 & 5 & 11 & 4 & 1 & 4 & 9 & 13 & 21 & 3 & 13 & 1 & 4 & 6 & 7 & 10 & 24 \\
\hline 10 & 12 & 4 & 2 & 3 & 8 & 5 & 22 & 12 & 2 & 14 & 3 & 2 & 11 & 2 & 16 & 18 \\
\hline 11 & 3 & 13 & 1 & 4 & 4 & 9 & 8 & 26 & 3 & 13 & 3 & 2 & 4 & 9 & 10 & 24 \\
\hline 12 & 6 & 10 & 2 & 3 & 6 & 7 & 14 & 20 & 3 & 13 & 1 & 4 & 8 & 5 & 12 & 22 \\
\hline 13 & 10 & 6 & 2 & 3 & 6 & 7 & 18 & 16 & 1 & 15 & 1 & 4 & 1 & 12 & 3 & 31 \\
\hline 14 & 10 & 6 & 5 & 0 & 6 & 7 & 21 & 13 & 2 & 14 & 2 & 3 & 3 & 10 & 7 & 27 \\
\hline 15 & 5 & 11 & 4 & 1 & 5 & 8 & 14 & 20 & 2 & 14 & 4 & 1 & 7 & 6 & 13 & 21 \\
\hline 16 & 2 & 14 & 2 & 3 & 4 & 9 & 8 & 26 & 2 & 14 & 3 & 2 & 2 & 11 & 7 & 27 \\
\hline 17 & 6 & 10 & 3 & 2 & 5 & 8 & 14 & 20 & 4 & 12 & 1 & 4 & 7 & 6 & 12 & 22 \\
\hline 18 & 2 & 14 & 3 & 2 & 7 & 6 & 12 & 22 & 9 & 7 & 4 & 1 & 6 & 7 & 19 & 15 \\
\hline 19 & 2 & 14 & 1 & 4 & 3 & 10 & 6 & 28 & 3 & 13 & 1 & 4 & 2 & 11 & 6 & 28 \\
\hline 20 & 8 & 8 & 1 & 4 & 3 & 10 & 12 & 22 & 2 & 14 & 2 & 3 & 1 & 12 & 5 & 29 \\
\hline 21 & 6 & 10 & 3 & 2 & 7 & 6 & 16 & 18 & 1 & 15 & 0 & 5 & 3 & 10 & 4 & 30 \\
\hline 22 & 3 & 13 & 2 & 3 & 6 & 7 & 11 & 23 & 1 & 15 & 2 & 3 & 7 & 6 & 10 & 24 \\
\hline 23 & 8 & 8 & 2 & 3 & 7 & 6 & 17 & 17 & 1 & 15 & 3 & 2 & 3 & 10 & 7 & 27 \\
\hline 24 & 4 & 12 & 1 & 4 & 4 & 9 & 9 & 25 & 2 & 14 & 3 & 2 & 5 & 8 & 10 & 24 \\
\hline 25 & 4 & 12 & 1 & 4 & 7 & 6 & 12 & 22 & 2 & 14 & 2 & 3 & 1 & 12 & 5 & 29 \\
\hline 26 & 12 & 4 & 3 & 2 & 8 & 5 & 23 & 11 & 5 & 11 & 3 & 2 & 3 & 10 & 11 & 23 \\
\hline 27 & 10 & 6 & 3 & 2 & 6 & 7 & 19 & 15 & 2 & 14 & 3 & 2 & 5 & 8 & 10 & 24 \\
\hline 28 & 7 & 9 & 2 & 3 & 7 & 6 & 16 & 18 & 2 & 14 & 4 & 1 & 7 & 6 & 13 & 21 \\
\hline 29 & 6 & 10 & 5 & 0 & 10 & 3 & 21 & 13 & 0 & 16 & 3 & 2 & 2 & 11 & 5 & 29 \\
\hline 30 & 3 & 13 & 3 & 2 & 8 & 5 & 14 & 20 & 4 & 12 & 2 & 3 & 5 & 8 & 11 & 23 \\
\hline 31 & 2 & 14 & 1 & 4 & 9 & 4 & 12 & 22 & 2 & 14 & 1 & 4 & 1 & 12 & 4 & 30 \\
\hline 32 & 6 & 10 & 4 & 1 & 9 & 4 & 19 & 15 & 5 & 11 & 2 & 3 & 9 & 4 & 16 & 18 \\
\hline 33 & 9 & 7 & 4 & 1 & 10 & 3 & 23 & 11 & 2 & 14 & 0 & 5 & 11 & 2 & 13 & 21 \\
\hline 34 & 8 & 8 & 2 & 3 & 8 & 5 & 18 & 16 & 7 & 9 & 3 & 2 & 2 & 11 & 12 & 22 \\
\hline 35 & 7 & 9 & 3 & 2 & 6 & 7 & 16 & 18 & 10 & 6 & 2 & 3 & 3 & 10 & 15 & 19 \\
\hline TOTAL & 220 & 340 & 97 & 78 & 238 & 217 & 555 & 635 & 112 & 448 & 76 & 99 & 159 & 296 & 347 & 843 \\
\hline ‘ MÉDIA & 6,3 & 9,7 & 2,8 & 2,2 & 6,8 & 6,2 & 15,9 & 18,1 & 3,2 & 12,8 & 2,2 & 2,8 & 4,5 & 8,5 & 9,9 & 24,1 \\
\hline$\%$ & 39,6 & 60,7 & 55,4 & 44,6 & 52,3 & 47,7 & 46,6 & 53,4 & 20,0 & 80,0 & 43,4 & 56,6 & 34,9 & 65,1 & 29,2 & 70,8 \\
\hline
\end{tabular}




\section{ANEXO 3}

Tabela 4 - Número e média de erro (E) e acerto (A), durante as fases de preparo do medicamento (PM), preparo do paciente (PP) e aplicação do medicamento (AM), dos sujeitos dos grupos controle e experimental ao realizarem o procedimento com medicamento contido em ampola (34 itens), em situação real 1.

\begin{tabular}{|c|c|c|c|c|c|c|c|c|c|c|c|c|c|c|c|c|}
\hline \multirow{3}{*}{ SUIEITOS } & \multicolumn{8}{|c|}{ GRUPO CONTROLE $(\mathrm{N}=27)$} & \multicolumn{8}{|c|}{ GRUPO EXPERIMENTAL $(N=25)$} \\
\hline & \multicolumn{2}{|c|}{ PM } & \multicolumn{2}{|l|}{ PP } & \multicolumn{2}{|l|}{ AM } & \multicolumn{2}{|c|}{ TOTAL } & \multicolumn{2}{|c|}{ PM } & \multicolumn{2}{|c|}{ PP } & \multicolumn{2}{|c|}{ AM } & \multicolumn{2}{|c|}{ TOTAL } \\
\hline & E & A & E & A & E & A & E & A & E & A & E & A & E & A & E & A \\
\hline 1 & 6 & 10 & 4 & 1 & 9 & $\overline{4}$ & 19 & 15 & 2 & 14 & 2 & 3 & 5 & 8 & 9 & 25 \\
\hline 2 & 13 & 3 & 4 & 1 & 7 & 6 & 24 & 10 & 2 & 14 & 0 & 5 & 5 & 8 & 7 & 27 \\
\hline 3 & 8 & 8 & 1 & 4 & 3 & 10 & 12 & 22 & 2 & 14 & 2 & 3 & 5 & 8 & 9 & 25 \\
\hline 4 & 1 & 15 & 2 & 3 & 8 & 5 & 11 & 23 & 4 & 12 & 3 & 2 & 2 & 11 & 9 & 25 \\
\hline 5 & 8 & 8 & 4 & 1 & 9 & 4 & 21 & 13 & 7 & 9 & 2 & 3 & 2 & 11 & 11 & 23 \\
\hline 6 & 3 & 13 & 4 & 1 & 13 & 0 & 20 & 14 & 3 & 13 & 2 & 3 & 5 & 8 & 10 & 24 \\
\hline 7 & 5 & 11 & 4 & 1 & 4 & 9 & 13 & 21 & 2 & 13 & 3 & 2 & 4 & 9 & 10 & 24 \\
\hline 8 & 12 & 4 & 2 & 3 & 8 & 5 & 22 & 12 & 3 & 13 & 1 & 4. & 8 & 5 & 12 & 22 \\
\hline 9 & 3 & 13 & 1 & 4 & 4 & 9 & 8 & 26 & 1 & 15 & 1 & 4 & 1 & 12 & 3 & 31 \\
\hline 10 & 6 & 10 & 2 & 3 & 6 & 7 & 14 & 20 & 2 & 14 & 2 & 3 & 3 & 10 & 7 & 27 \\
\hline 11 & 10 & 6 & 2 & 3 & 6 & 7 & 18 & 16 & 2 & 14 & 3 & 2 & 2 & 11 & 7 & 27 \\
\hline 12 & 5 & 11 & 4 & 1 & 5 & 8 & 14 & 20 & 3 & 13 & 1 & 4 & 2 & 11 & 6 & 28 \\
\hline 13 & 2 & 14 & 2 & 3 & 4 & 9 & 8 & 26 & 2 & 14 & 2 & 3 & 1 & 12 & 5 & 29 \\
\hline 14 & 6 & 10 & 3 & 2 & 5 & 8 & 14 & 20 & 1 & 15 & 0 & 5 & 3 & 10 & 4 & 30 \\
\hline 15 & 2 & 14 & 3 & 2 & 7 & 6 & 12 & 22 & 1 & 15 & 2 & 3 & 7 & 6 & 10 & 24 \\
\hline 16 & 2 & 14 & 1 & 4 & 3 & 10 & 6 & 28 & 1 & 15 & 3 & 2 & 3 & 10 & 7 & 27 \\
\hline 17 & 6 & 10 & 3 & 2 & 7 & 6 & 16 & 18 & 2 & 14 & 3 & 2 & 5 & 8 & 10 & 24 \\
\hline 18 & 4 & 12 & 1 & 4 & 4 & 9 & 9 & 25 & 2 & 14 & 2 & 3 & 1 & 12 & 5 & 29 \\
\hline 19 & 4 & 12 & 1 & 4 & 7 & 6 & 12 & 22 & 2 & 14 & 4 & 1 & 7 & 6 & 13 & 21 \\
\hline 20 & 12 & 4 & 3 & 2 & 8 & 5 & 23 & 11 & 0 & 16 & 3 & 2 & 2 & 11 & 5 & 29 \\
\hline 21 & 10 & 6 & 3 & 2 & 6 & 7 & 19 & 15 & 4 & 12 & 2 & 3 & 5 & 8 & 11 & 23 \\
\hline 22 & 7 & 9 & 2 & 3 & 7 & 6 & 16 & 18 & 2 & 14 & 1 & 4 & 1 & 12 & 4 & 30 \\
\hline 23 & 3 & 13 & 3 & 2 & 8 & 5 & 14 & 20 & 2 & 14 & 0 & 5 & 11 & 2 & 13 & 21 \\
\hline 24 & 2 & 14 & 1 & 4 & 9 & 4 & 12 & 22 & 7 & 9 & 3 & 2 & 2 & 11 & 12 & 22 \\
\hline 25 & 6 & 10 & 4 & 1 & 9 & 4 & 19 & 15 & 10 & 6 & 2 & 3 & 3 & 10 & 15 & 19 \\
\hline 26 & 9 & 7 & 4 & 1 & 10 & 3 & 23 & 11 & - & . & 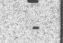 & - & - & - & - & 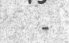 \\
\hline 27 & 8 & 8 & 2 & 3 & 8 & 5 & 18 & 16 & - & - & - & - & - & - & - & - \\
\hline TOTAL & 163 & 269 & 70 & 65 & 184 & 167 & 417 & 501 & 70 & 330 & 49 & 76 & 95 & 230 & 214 & 636 \\
\hline MÉDIA & 6,0 & 10,0 & 2,6 & 2,4 & 6,8 & 6,2 & 15,4 & 18,5 & 2,8 & 13,2 & 2,0 & 3,0 & 3,8 & 9,2 & 8,6 & 25,4 \\
\hline$\%$ & 37,7 & 62,3 & 51,9 & 48,1 & 52,4 & 47,6 & 45,4 & 54,6 & 17,5 & 82,5 & 39,2 & 60,8 & 29,2 & 70,8 & 25,2 & 74,8 \\
\hline
\end{tabular}

Total $=34$ itens; $P M=16$ itens; $A M=13$ itens 


\section{ANEXO 4}

Tabela 5 - Número e média de erro (E) e acerto (A), durante as fases de preparo do medicamento (PM), preparo do paciente (PP) e aplicação do medicamento (AM), dos sujeitos dos grupos controle e experimental ao realizarem o procedimento com medicamento contido em frasco ampola (46 itens), em situação real 1.

\begin{tabular}{|c|c|c|c|c|c|c|c|c|c|c|c|c|c|c|c|c|}
\hline \multirow{3}{*}{ SUJEITOS } & \multicolumn{8}{|c|}{ GRUPO CONTROLE $(\mathrm{N}=8)$} & \multicolumn{8}{|c|}{ GRUPO EXPERIMENTAL $(\mathrm{N}=10)$} \\
\hline & \multicolumn{2}{|c|}{ PM } & \multicolumn{2}{|c|}{ PP } & \multicolumn{2}{|c|}{ AM } & \multicolumn{2}{|c|}{ TOTAL } & \multicolumn{2}{|c|}{$P M$} & \multicolumn{2}{|c|}{$P P$} & \multicolumn{2}{|c|}{ AM } & \multicolumn{2}{|c|}{ TOTAL } \\
\hline & $E$ & A & $E$ & A & $E$ & A & $E$ & A & $E$ & A & $E$ & A & $E$ & A & $E=$ & A \\
\hline 1 & 16 & 12 & 4 & 1 & 8 & 5 & 28 & 18 & 9 & 19 & 1 & 4 & 2 & 11 & 12 & 34 \\
\hline 2 & 21 & 7 & 5 & 0 & 8 & 5 & 34 & 12 & 14 & 14 & 5 & 0 & 8 & 5 & 27 & 19 \\
\hline 3 & 19 & 9 & 5 & 0 & 6 & 7 & 30 & 16 & 11 & 17 & 1 & 4 & 6 & 7 & 18 & 28 \\
\hline 4 & 17 & 11 & 1 & 4 & 3 & 10 & 21 & 25 & 4 & 24 & 3 & 2 & 11 & 2 & 18 & 28 \\
\hline 5 & 5 & 23 & 2 & 3 & 6 & 7 & 13 & 33 & 5 & 22 & 4 & 1 & 7 & 6 & 17 & 29 \\
\hline 6 & 14 & 14 & 2 & 3 & 7 & 6 & 23 & 23 & 5 & 23 & 1 & 4 & 7 & 6 & 13 & 33 \\
\hline 7 & 10 & 18 & 5 & 0 & 10 & 3 & 25 & 21 & 16 & 12 & 4 & 1 & 6 & 7 & 26 & 20 \\
\hline 8 & 17 & 11 & 3 & 2 & 6 & 7 & 26 & 20 & 11 & 17 & 3 & 2 & 3 & 10 & 17 & 29 \\
\hline 9 & - & - & - & - & - & - & - & - & 10 & 18 & 3 & 2 & 5 & 8 & 18 & 28 \\
\hline 10 & - & - & - & - & - & - & - & - & 13 & 15 & 2 & 3 & 9 & 4 & 24 & 22 \\
\hline TOTAL & 119 & 105 & 27 & 13 & 54 & 50 & 200 & 168 & 99 & 181 & 27 & 23 & 64 & 66 & 190 & 270 \\
\hline MÉDIA & 14,9 & 13,1 & 3,4 & 1,6 & 6,8 & 6,3 & 25,0 & 21,0 & 9,9 & 18,1 & 2,7 & 2,3 & 6,4 & 6,6 & 19,0 & 27,0 \\
\hline$\%$ & 53,1 & 46,9 & 67,5 & 32,5 & 51,9 & 48,1 & 54,3 & 45,7 & 35,4 & 64,6 & 54,0 & 46,0 & 49,2 & 50,8 & 41,3 & 58,7 \\
\hline
\end{tabular}

Total $=46$ itens; $P M=28$ itens; $P P=5$ itens, $A M=13$ itens 


\section{REFERÊNCIAS BIBLIOGRÁFICAS}

1. ANGELO, M. Vivendo uma prova de fogo: as experiências iniciais da aula de enfermagem. São Paulo, 1989. 133p. Tese (Doutorado) - Instituto de Psicologia, Universidade de São Paulo.

2. BLOOM, B.S. Características humanas e aprendizagem escolar. Trad. De Maria Angela Vinagre de Almeida. Porto Alegre. Rio de Janeiro: Globo, 1981, cap.1. p.1-17: As diferenças individuais nos alunos e a aprendizagem.

3. EMBURRY, S.B.; THRUSTON, N.B. A preliminary study on the effect of role modeling on anxiety in motor skill performance and the effect of self concept in motor skill performance. Nurs.Papers, v.8, n.9, p.33-9, 1976.

4. FRENCH, P. A place for simulation in nurse education. Nurse Focus, v.1, n.11, p.445-6, 1980.

5. FRIEDLANDER, M.R. $O$ ensino de procedimentos básicos no laboratório de enfermagem: comparação entre dois métodos de instrução. São Paulo, 1984b. 142p. Tese (Doutorado). Escola de Enfermagem de São Paulo, Universidade de São Paulo.

7. GRIGSBY, K.; SMITH, S. Teaching basic nursing skills in a new curriculum. J.Nurs.Educ. v.16, n.3, p.17-21, 1977.

8. HORR, L.; FARIA, E.M.; CAMARGO, A.P.S.; ORO, I.M.; SOUZA, M.L. Determinação do rendimento instrumental da ministração de medicamentos por via intramuscular em pacientes hospitalizados. $R$. Bras. Enferm., v.31, n.4, p.478-95, 1978.

9. INFANTE, M.S. Toward effective and effecient use of the clinical laboratory. Nurse Educ. v.6, p.16-9, 1931.

10. MIYADAHIRA, A.M.K. Processo de ensino-aprendizagem de habilidades psicomotoras: análise da técnica de injeção intramuscular. São Paulo, 1990, 243p. Tese(Doutorado) - Escola de Enfermagem de São Paulo, Universidade de São Paulo.

11. NOGUEIRA, M.S. Aplicaçāo de injetáveis via intramuscular. comparação entre dois métodos de ensino. Ribeirão Preto, 1995, 154p. Tese (Doutorado) - Escola de Enfermagem de Ribeirão Preto, Universidade de São Paulo.

12. PENTEADO, W.M.A. O modelo de ensino para o domínio. In: et al Psicologia e ensino. São Paulo, Papelibros, 1980. p. 168-179.

13. SHALLOWHORN, G. Intramuscular injection. Am.J.Nurs., v.54, n.4, p.43841, 1954.

14. SIEGEL, S. Estatística não paramétrica para as ciências do comportamento. São Paulo: Mc Graw-Hill, 1975. 